\title{
CORRELAÇÃO ENTRE O ERRO EXPERIMENTAL E CARACTERÍSTICAS DOS ENSAIOS NACIONAIS DE COMPETIÇÃO DE CULTIVARES DE MILHO
}

\section{CORRELATION BETWEEN THE EXPERIMENTAL ERROR AND CHARACTERISTICS OF THE NATIONAL COMPETITION EXPERIMENTS OF MAIZE CULTIVARS}

\author{
Alessandro Dal'Col Lúcio ${ }^{1}$ David Ariovaldo Banzatto ${ }^{2}$ Lindolfo Storck $^{3}$ \\ Thomas Newton Martin ${ }^{4}$ Leandro Homrich Lorentz ${ }^{5}$
}

RESUMO

\begin{abstract}
O objetivo deste trabalho foi verificar o grau de relação entre o erro experimental e as variáveis observadas no decorrer do experimento. Com os dados dos ensaios nacionais de competição de cultivares de milho, utilizou-se a análise de trilha dentro de cada ciclo dos materiais, tendo como variável principal o quadrado médio do erro e como variáveis explicativas as demais variáveis observadas nos experimentos, com o objetivo de mostrar que variáveis como peso de espigas, rendimento de grãos, altura da planta e altura de inserção da primeira espiga com valores mais homogêneos dentro de cada experimento, independente do ciclo, proporcionam um menor valor do quadrado médio do erro, fazendo com que a precisão do experimento seja melhorada.
\end{abstract}

Palavras-chave: precisão experimental, análise de trilha, Zea mays.

\section{SUMMARY}

The objective of the work was to verify the relationship between the experimental error and the variable observed in the time course of the experiment. With the results of the national competition experiments of maize cultivars, pathy analysis was used inside of each cycle, having as dependent variable the mean square of the error and as independent variables the other variables observed in the experiments, showing that the variables as weight of spikes, grain yield, height of the plant and insertion of the first spikes with more homogeneous values inside of each experiment, disregarding the cycle, provided a smaller value of the mean square of the error, improving the precision of the experiment.

Key words: experimental precision, pathy analysis, Zea mays.

\section{INTRODUÇÃO}

Devido à importância da cultura do milho no Brasil, várias pesquisas são realizadas para exploração do potencial produtivo desta cultura. $\mathrm{O}$ Centro Nacional de Pesquisa de Milho e Sorgo/Embrapa coordena o Ensaio Nacional de Cultivares de Milho, onde anualmente são distribuídos aos pesquisadores cerca de 230 experimentos que formam a rede de ensaio nacional, sendo destes, 35, 35 e 30\% de ciclos Precoce, Super precoce e Normal, respectivamente, com o objetivo de avaliar cultivares de milho em comercialização ou em vias de lançamento. Os resultados dessa avaliação são utilizados como suporte para elaboração de uma lista de recomendação de cultivares de milho para as diferentes regiões do país (EMBRAPA, 1997).

LÚCIO (1997), analisando resultados da avaliação desses cultivares, percebeu a existência de alguns com precisão inadequada, pois certos experimentos apresentaram valores da diferença mínima significativa, para os teste de comparação de médias, superiores à média obtida entre as cultivares de milho cultivadas em campo de produção. Essa baixa precisão, normalmente, resulta em ineficientes discriminações entre os tratamentos (híbridos de milho), induzindo a conclusões incorretas ao se

\footnotetext{
${ }^{1}$ Engenheiro Agrônomo, Doutor, Professor Adjunto, Departamento de Fitotecnia (DF), Centro de Ciências Rurais (CCR), Universidade Federal de Santa Maria (UFSM). 97115-900, Santa Maria, RS. E-mail: adlucio@ ccr.ufsm.br. Autor para correspondência.

${ }^{2}$ Engenheiro Agrônomo, Doutor, Professor Aposentado, Departamento de Ciências Exatas, Faculdade de Ciências Agrárias e Veterinárias, Universidasde Estadual Paulista. Jaboticabal, SP. E-mail: banzatto@asbyte.com.br.

${ }^{3}$ Engenheiro Agrônomo, Doutor, Professor Titular, DF/CCR/UFSM, Santa Maria, RS, bolsista CNPq. E-mail: storck@ccr.ufsm.br.

${ }^{4}$ Aluno do Curso de Graduação em Agronomia, CCR/UFSM, bolsista CNPq.

${ }^{5}$ Aluno do Curso de Graduação em Agronomia, CCR/UFSM, bolsista FAPERGS.
} 
fazerem as recomendações aos produtores. A falta de controle de qualidade dos experimentos de milho resulta em prejuízos muitas vezes incalculáveis e significativos economicamente.

Segundo autores como STEEL et al (1996), NETER \& WASSERMAN (1999), FEDERER (1977), HINKELMANN \& KEMPTHORNE (1994), BANZATTO \& KRONKA (1995) e STORCK et al. (2000), o uso dos princípios básicos da experimentação no planejamento, condução e análise de experimentos, é fundamental para se manter o erro experimental em níveis aceitáveis. Outras formas de minimizar o efeito do erro experimental na qualidade de experimentos são o uso de maior número de repetições, combinado com parcelas menores (GOMES, 1994; CONAGIN et $\boldsymbol{a l} ., 1995$ e STORCK $\boldsymbol{e t}$ al., 2000) e a realização o mais uniforme possível dos tratos culturais inerentes à cultura, no decorrer do experimento (LOPES, 1993; LOPES \& STORCK, 1995; LÚCIO, 1997). Estes autores mostram que tratos culturais, como aplicação de inseticidas e adubações desuniformes, são capazes de aumentar o erro experimental. Já o desbaste, distribuição manual de sementes na linha de semeadura e controle de ervas daninhas são capazes de reduzir o erro para experimentos com a cultura do milho.

Assim, o presente trabalho teve por objetivo estimar o grau de relação entre o erro experimental e as variáveis observadas no decorrer de experimentos de competição de cultivares de milho.

\section{MATERIAL E MÉTODOS}

O material utilizado refere-se aos resultados dos 522 experimentos em Rede de Ensaios Nacionais de Competição de Cultivares de Milho, realizados entre os anos de 1986 e 1996, para materiais de ciclo normal, precoce e superprecoce. Estes resultados foram obtidos junto ao Centro Nacional de Pesquisa de Milho e Sorgo/EMBRAPA.

Em cada experimento, foram utilizadas as seguintes variáveis: ciclo do material (super precoce, precoce e normal); número de tratamentos (I); coeficiente de variação (CV\%) da variável peso de grãos, e média por tratamento para: peso de espiga, em $\mathrm{kg} / \mathrm{ha}$; peso de grãos, em $\mathrm{kg} / \mathrm{ha}$; dias até o florescimento; altura da planta, em $\mathrm{cm}$; altura de inserção da primeira espiga, em cm; percentagem de plantas acamadas; percentagem de plantas quebradas; estande final; número de espigas.

Os experimentos foram devidamente catalogados, e os resultados de cada um compuseram um arquivo a partir dos quais, utilizando-se de um programa em Turbo Pascal, calcularam-se médias e variâncias entre as médias de tratamentos, que estimam os quadrados médios de tratamento, para cada variável estudada no experimento, além do quadrado médio do erro (QMe $=\frac{\mathrm{CV}^{2} \times \overline{\mathrm{X}^{2}}}{100^{2}}$ ) para a variável peso de grãos, criando-se um novo e único arquivo, com estes resultados.

Para esclarecimento do grau de relação direta entre o QMe e a média e variância das características avaliadas nos ensaios, e entre essas características, foi realizada a análise de correlação de Pearson com os resultados obtidos, para cada ciclo individualmente. O coeficiente de correlação de Pearson, segundo COSTA NETO (1977), é de grande utilidade na quantificação da magnitude e direção das influências de fatores, mas não dá a exata importância relativa dos efeitos diretos e indiretos das variáveis independentes sobre a dependente, pois a correlação é apenas uma medida de associação. Assim, realizou-se uma análise de trilha (VENCOVSKY \& BARRIGA, 1992; CRUZ \& REGAZZI, 1994) para cada ciclo, estudando-se os efeitos diretos e indiretos das variáveis utilizadas, sobre a variável dependente QMe, através das estimativas e interpretações dos coeficientes de trilha, definido como a relação entre o desvio padrão do efeito e o desvio padrão total do efeito, medindo a influência direta de uma variável sobre a outra independente das demais variáveis e a influência indireta através das demais variáveis avaliadas. Para a análise de trilha, utilizou-se o software GENES (CRUZ, 1997).

\section{RESULTADOS E DISCUSSÃO}

A média dos QMe para o peso de grãos foi igual a $0,5645,0,6112$ e 0,5349 , respectivamente para experimentos de ciclo superprecoce, precoce e normal e, para os mesmos experimentos, a média geral do peso de grãos foi de $6,69,6,35$ e 5,99t/ha com coeficiente de variação médio de $10,85,12,38$ e $12,49 \%$ respectivamente, classificados por LÚCIO (1997) como médios, dentro de cada ciclo.

Os resultados da análise de trilha, para cada ciclo de material de milho, estão apresentados nas tabelas 1, 2 e 3. Apesar das baixas correlações de Pearson apresentadas, o desdobramento destas em efeito direto e indireto mostrou algumas relações de interesse, levando em conta alguns pontos essenciais da relação entre o coeficiente de correlação de Pearson e o seu efeito direto. Quando estes forem iguais ou semelhantes, em magnitude e sinal, esta 
Tabela 1 - Coeficientes de correlação parcial e de Pearson, devidos aos efeitos direto e indireto das variáveis observadas nos 117 experimentos de cultivares de milho de ciclo superprecoce, sobre a estatística quadrado médio do erro (QMe). Santa Maria - RS, 2000.

\begin{tabular}{|c|c|c|c|c|c|c|c|c|c|c|c|c|c|c|c|c|c|c|}
\hline \multirow{2}{*}{ Efeito } & \multicolumn{18}{|c|}{ Variável ${ }^{1}$} \\
\hline & Mpesp & Vpesp & Mpg & Vpg & Mfl & Vfl & Maltpl & Valtpl & Maltesp & Valtesp & Mpac & Vpac & Mpqb & Vpqb & Mef & Vef & Mnesp & Vnesp \\
\hline Direito & $0,37 *$ & 0,09 & $0,44 *$ & 0,00 & 0,00 & 0,00 & 0,00 & 0,00 & 0,00 & 0,00 & 0,00 & 0,00 & 0,00 & 0,00 & 0,00 & 0,00 & 0,00 & 0,00 \\
\hline Mpesp & - & $0,25^{*}$ & $-0,00$ & 0,00 & 0,00 & 0,00 & 0,00 & 0,00 & 0,00 & 0,00 & 0,00 & 0,00 & 0,00 & 0,00 & 0,00 & 0,00 & 0,00 & 0,00 \\
\hline Vpesp & 0,06 & - & 0,01 & 0,00 & 0,00 & 0,00 & 0,00 & 0,00 & 0,00 & 0,00 & 0,00 & 0,00 & 0,00 & 0,00 & 0,00 & 0,00 & 0,00 & 0,00 \\
\hline Mpg & $-0,00$ & 0,04 & - & 0,00 & 0,00 & 0,00 & 0,00 & 0,00 & 0,00 & 0,00 & 0,00 & 0,00 & 0,00 & 0,00 & 0,00 & 0,00 & 0,00 & 0,00 \\
\hline Vpg & 0,00 & 0,00 & 0,00 & - & 0,00 & 0,00 & 0,00 & 0,00 & 0,00 & 0,00 & 0,00 & 0,00 & 0,00 & 0,00 & 0,00 & 0,00 & 0,00 & 0,00 \\
\hline Mfl & 0,00 & 0,00 & 0,00 & 0,00 & - & 0,00 & 0,00 & 0,00 & 0,00 & 0,00 & 0,00 & 0,00 & 0,00 & 0,00 & 0,00 & 0,00 & 0,00 & 0,00 \\
\hline Vfl & 0,00 & 0,00 & 0,00 & 0,00 & 0,00 & - & 0,00 & 0,00 & 0,00 & 0,00 & 0,00 & 0,00 & 0,00 & 0,00 & 0,00 & 0,00 & 0,00 & 0,00 \\
\hline Maltpl & 0,00 & 0,00 & 0,00 & 0,00 & 0,00 & 0,00 & - & 0,00 & 0,00 & 0,00 & 0,00 & 0,00 & 0,00 & 0,00 & 0,00 & 0,00 & 0,00 & 0,00 \\
\hline Valtpl & 0,00 & 0,00 & 0,00 & 0,00 & 0,00 & 0,00 & 0,00 & - & 0,00 & 0,00 & 0,00 & 0,00 & 0,00 & 0,00 & 0,00 & 0,00 & 0,00 & 0,00 \\
\hline Maltesp & 0,00 & 0,00 & 0,00 & 0,00 & 0,00 & 0,00 & 0,00 & 0,00 & - & 0,00 & 0,00 & 0,00 & 0,00 & 0,00 & 0,00 & 0,00 & 0,00 & 0,00 \\
\hline Valtesp & 0,00 & 0,00 & 0,00 & 0,00 & 0,00 & 0,00 & 0,00 & 0,00 & 0,00 & - & 0,00 & 0,00 & 0,00 & 0,00 & 0,00 & 0,00 & 0,00 & 0,00 \\
\hline Mpac & 0,00 & 0,00 & 0,00 & 0,00 & 0,00 & 0,00 & 0,00 & 0,00 & 0,00 & 0,00 & - & 0,00 & 0,00 & 0,00 & 0,00 & 0,00 & 0,00 & 0,00 \\
\hline Vpac & 0,00 & 0,00 & 0,00 & 0,00 & 0,00 & 0,00 & 0,00 & 0,00 & 0,00 & 0,00 & 0,00 & - & 0,00 & 0,00 & 0,00 & 0,00 & 0,00 & 0,00 \\
\hline Mpqb & 0,00 & 0,00 & 0,00 & 0,00 & 0,00 & 0,00 & 0,00 & 0,00 & 0,00 & 0,00 & 0,00 & 0,00 & - & 0,00 & 0,00 & 0,00 & 0,00 & 0,00 \\
\hline Vpqb & 0,00 & 0,00 & 0,00 & 0,00 & 0,00 & 0,00 & 0,00 & 0,00 & 0,00 & 0,00 & 0,00 & 0,00 & 0,00 & - & 0,00 & 0,00 & 0,00 & 0,00 \\
\hline Mef & 0,00 & 0,00 & 0,00 & 0,00 & 0,00 & 0,00 & 0,00 & 0,00 & 0,00 & 0,00 & 0,00 & 0,00 & 0,00 & 0,00 & - & 0,00 & 0,00 & 0,00 \\
\hline Vef & 0,00 & 0,00 & 0,00 & 0,00 & 0,00 & 0,00 & 0,00 & 0,00 & 0,00 & 0,00 & 0,00 & 0,00 & 0,00 & 0,00 & 0,00 & - & 0,00 & 0,00 \\
\hline Mnesp & 0,00 & 0,00 & 0,00 & 0,00 & 0,00 & 0,00 & 0,00 & 0,00 & 0,00 & 0,00 & 0,00 & 0,00 & 0,00 & 0,00 & 0,00 & 0,00 & - & 0,00 \\
\hline Vnesp & 0,00 & 0,00 & 0,00 & 0,00 & 0,00 & 0,00 & 0,00 & 0,00 & 0,00 & 0,00 & 0,00 & 0,00 & 0,00 & 0,00 & 0,00 & 0,00 & 0,00 & - \\
\hline Pearson & 0,08 & 0,05 & $0,18^{*}$ & 0,00 & 0,00 & 0,00 & 0,00 & 0,00 & 0,00 & 0,00 & 0,00 & 0,00 & 0,00 & 0,00 & 0,00 & 0,00 & 0,00 & 0,00 \\
\hline
\end{tabular}

${ }^{1} \mathrm{M}$ e V indicam, respectivamente, média e variância das variáveis: peso de espiga, peso de grãos, dias até florescimento, altura de planta, altura de inserção da primeira espiga, número de plantas acamadas, número de plantas quebradas, estande final e número de espigas;

*: Significativo em nível de $5 \%$ de probabilidade de erro.

correlação direta explica a verdadeira associação existente. Se o coeficiente de correlação de Pearson for positivo, mas o efeito direto for negativo ou desprezível, a correlação será indicada pelos efeitos indiretos, sendo estes considerados na análise. Com o coeficiente de correlação de Pearson desprezível e o efeito direto apresentando-se positivo e alto, os efeitos indiretos é que são responsáveis pela falta de correlação, merecendo a mesma atenção na análise. Finalmente, com a correlação de Pearson negativa e efeito direto positivo e alto, devem-se eliminar os efeitos indiretos da análise e aproveitar somente os diretos (VENCOVSKY \& BARRIGA, 1992).

Independente do ciclo avaliado, o comportamento das variáveis média e variância do peso de espigas (Mpesp e Vpesp) e média do peso de grãos (Mpg) mostrou-se semelhante, apresentando valores significativos e positivos para os coeficientes de Pearson e para os seus efeitos diretos com o QMe do peso de grãos (Tabelas 1, 2 e 3), fazendo com que a relação entre estas variáveis e este valor do QMe, deva ser considerada principalmente na escolha dos materiais que irão compor os tratamentos, dentro de cada ciclo, pois a medida que se aumentam as médias do peso de espigas e do peso de grãos, bem como a variância do peso de espigas, há uma redução na precisão experimental. Isto não indica que deverão ser selecionados materiais com menor potencial produtivo e sim materiais mais homogêneos, ou seja, que realmente possuam potenciais produtivos semelhantes, reduzindo assim a variância do peso de espigas e também do peso de grãos, reduzindo assim o erro experimental.

Seguindo a interpretação sugerida por VENCOVSKY \& BARRIGA (1992), a relação entre o coeficiente de Pearson e os efeitos direto da Mpesp com o QMe do peso de grãos nos experimentos de ciclo super precoce (Tabela 1) mostra que os efeitos indiretos dessa variável devem ser considerados, ou seja, existe uma relação indireta da média do peso de espigas com as demais variáveis observadas no experimento, fazendo com que exista uma relação direta entre Mpesp e QMe. Este mesmo comportamento está presente na variável Vpesp, indicando que as outras variáveis exercem uma interferência indireta no QMe do peso de grãos, pois a medida que se aumenta, por exemplo, o valor da média de plantas acamadas e de plantas quebradas, há um acréscimo no valor do QMe. Assim, para se obterem menores valores do QMe para peso de grãos, deve-se realizar uma seleção de materiais adequada para o experimento, fazendo com que a variabilidade entre eles seja baixa, independente da variável, reduzindo assim o quadrado médio de tratamento na análise da variância e, por consequiência, o quadrado médio do erro.

Na tabela 3, outras variáveis apresentaram relações significativas. Pode-se observar que a média da altura das plantas (Maltpl) dos materiais de 
Tabela 2 - Coeficientes de correlação parcial e de Pearson, devidos aos efeitos direto e indireto das variáveis observadas nos 207 experimentos de cultivares de milho de ciclo precoce, sobre a estatística quadrado médio do erro (QMe). Santa Maria - RS, 2000.

\begin{tabular}{|c|c|c|c|c|c|c|c|c|c|c|c|c|c|c|c|c|c|c|}
\hline \multirow{2}{*}{ Efeito } & \multicolumn{18}{|c|}{ Variável $^{1}$} \\
\hline & Mpesp & Vpesp & Mpg & Vpg & Mfl & Vfl & Maltpl & Valtpl & Maltesp & Valtesp & Mpac & Vpac & Mpqb & Vpqb & Mef & Vef & Mnesp & Vnesp \\
\hline Direito & $0,52 *$ & $0,25^{*}$ & $0,60 *$ & 0,00 & 0,00 & 0,00 & 0,00 & 0,00 & 0,00 & 0,00 & 0,00 & 0,00 & 0,00 & 0,00 & 0,00 & 0,00 & 0,00 & 0,00 \\
\hline Mpesp & - & $0,37^{*}$ & 0,11 & 0,00 & 0,00 & 0,00 & 0,00 & 0,00 & 0,00 & 0,00 & 0,00 & 0,00 & 0,00 & 0,00 & 0,00 & 0,00 & 0,00 & 0,00 \\
\hline Vpesp & $0,18^{*}$ & - & 0,04 & 0,00 & 0,00 & 0,00 & 0,00 & 0,00 & 0,00 & 0,00 & 0,00 & 0,00 & 0,00 & 0,00 & 0,00 & 0,00 & 0,00 & 0,00 \\
\hline Mpg & 0,13 & 0,11 & - & 0,00 & 0,00 & 0,00 & 0,00 & 0,00 & 0,00 & 0,00 & 0,00 & 0,00 & 0,00 & 0,00 & 0,00 & 0,00 & 0,00 & 0,00 \\
\hline Vpg & 0,00 & 0,00 & 0,00 & - & 0,00 & 0,00 & 0,00 & 0,00 & 0,00 & 0,00 & 0,00 & 0,00 & 0,00 & 0,00 & 0,00 & 0,00 & 0,00 & 0,00 \\
\hline Mfl & 0,00 & 0,00 & 0,00 & 0,00 & - & 0,00 & 0,00 & 0,00 & 0,00 & 0,00 & 0,00 & 0,00 & 0,00 & 0,00 & 0,00 & 0,00 & 0,00 & 0,00 \\
\hline Vfl & 0,00 & 0,00 & 0,00 & 0,00 & 0,00 & - & 0,00 & 0,00 & 0,00 & 0,00 & 0,00 & 0,00 & 0,00 & 0,00 & 0,00 & 0,00 & 0,00 & 0,00 \\
\hline Maltpl & 0,00 & 0,00 & 0,00 & 0,00 & 0,00 & 0,00 & - & 0,00 & 0,00 & 0,00 & 0,00 & 0,00 & 0,00 & 0,00 & 0,00 & 0,00 & 0,00 & 0,00 \\
\hline Valtpl & 0,00 & 0,00 & 0,00 & 0,00 & 0,00 & 0,00 & 0,00 & - & 0,00 & 0,00 & 0,00 & 0,00 & 0,00 & 0,00 & 0,00 & 0,00 & 0,00 & 0,00 \\
\hline Maltesp & 0,00 & 0,00 & 0,00 & 0,00 & 0,00 & 0,00 & 0,00 & 0,00 & - & 0,00 & 0,00 & 0,00 & 0,00 & 0,00 & 0,00 & 0,00 & 0,00 & 0,00 \\
\hline Valtesp & 0,00 & 0,00 & 0,00 & 0,00 & 0,00 & 0,00 & 0,00 & 0,00 & 0,00 & - & 0,00 & 0,00 & 0,00 & 0,00 & 0,00 & 0,00 & 0,00 & 0,00 \\
\hline Mpac & 0,00 & 0,00 & 0,00 & 0,00 & 0,00 & 0,00 & 0,00 & 0,00 & 0,00 & 0,00 & - & 0,00 & 0,00 & 0,00 & 0,00 & 0,00 & 0,00 & 0,00 \\
\hline Vpac & 0,00 & 0,00 & 0,00 & 0,00 & 0,00 & 0,00 & 0,00 & 0,00 & 0,00 & 0,00 & 0,00 & - & 0,00 & 0,00 & 0,00 & 0,00 & 0,00 & 0,00 \\
\hline Mpqb & 0,00 & 0,00 & 0,00 & 0,00 & 0,00 & 0,00 & 0,00 & 0,00 & 0,00 & 0,00 & 0,00 & 0,00 & - & 0,00 & 0,00 & 0,00 & 0,00 & 0,00 \\
\hline $\mathrm{Vpqb}$ & 0,00 & 0,00 & 0,00 & 0,00 & 0,00 & 0,00 & 0,00 & 0,00 & 0,00 & 0,00 & 0,00 & 0,00 & 0,00 & - & 0,00 & 0,00 & 0,00 & 0,00 \\
\hline Mef & 0,00 & 0,00 & 0,00 & 0,00 & 0,00 & 0,00 & 0,00 & 0,00 & 0,00 & 0,00 & 0,00 & 0,00 & 0,00 & 0,00 & - & 0,00 & 0,00 & 0,00 \\
\hline Vef & 0,00 & 0,00 & 0,00 & 0,00 & 0,00 & 0,00 & 0,00 & 0,00 & 0,00 & 0,00 & 0,00 & 0,00 & 0,00 & 0,00 & 0,00 & - & 0,00 & 0,00 \\
\hline Mnesp & 0,00 & 0,00 & 0,00 & 0,00 & 0,00 & 0,00 & 0,00 & 0,00 & 0,00 & 0,00 & 0,00 & 0,00 & 0,00 & 0,00 & 0,00 & 0,00 & - & 0,00 \\
\hline Vnesp & 0,00 & 0,00 & 0,00 & 0,00 & 0,00 & 0,00 & 0,00 & 0,00 & 0,00 & 0,00 & 0,00 & 0,00 & 0,00 & 0,00 & 0,00 & 0,00 & 0,00 & - \\
\hline Pearson & $0,15^{*}$ & $0,26^{*}$ & $0,19^{*}$ & 0,00 & 0,00 & 0,00 & 0,00 & 0,00 & 0,00 & 0,00 & 0,00 & 0,00 & 0,00 & 0,00 & 0,00 & 0,00 & 0,00 & 0,00 \\
\hline
\end{tabular}

${ }^{1} \mathrm{M}$ e V indicam, respectivamente, média e variância das variáveis: peso de espiga, peso de grãos, dias até florescimento, altura de planta, altura de inserção da primeira espiga, número de plantas acamadas, número de plantas quebradas, estande final e número de espigas;

*: Significativo em nível de $5 \%$ de probabilidade de erro.

ciclo normal, apresentou os coeficiente de Pearson e os efeitos diretos significativos e negativos, mostrando que a medida que aumenta esta média, o QMe do peso de grãos reduz, ou seja, o uso de plantas mais altas no experimento, é favorável à redução do erro experimental, pois são plantas mais vigorosas, tendo condições de expressar seu potencial produtivo. Já a variância da altura da planta (Valtpl) possui os valores dos coeficiente positivos e significativos, comprovando que a escolha de materiais mais uniformes para compor os tratamentos, favorece a redução do erro experimental.

Para a média da altura de inserção da primeira espiga (Maltesp) para os materiais de ciclo normal (Tabela 3), também a medida que se aumentam os valores desta variável, há uma redução no erro experimental, ou seja, experimentos que apresentam, em média, plantas com boa altura de inserção da primeira espiga, tendem a ser mais precisos, pois haverá menores problemas de formação da espiga.

Da mesma forma apresentada pela Valtpl, a variância da altura de inserção da primeira espiga mostrou coeficientes positivos e significativos (Tabela 3), mostrando que experimentos com alta variabilidade na altura de inserção da primeira espiga possuem maiores valores do QMe para peso de grãos.
Observa-se que, independente do ciclo do material avaliado, o comportamento das variáveis sobre o QMe do peso de grãos segue a mesma tendência de que quanto maior variabilidade na variável estudada, maior será o valor do QMe, reduzindo assim a precisão experimental. Assim, a definição dos materiais que irão compor os tratamentos torna-se de fundamental importância no experimento, principalmente na real definição do ciclo do mesmo, favorecendo uma maior homogeneidade entre os materiais avaliados, dentro de cada ciclo e para cada variável observada, sem contudo, reduzir a média de produtividade de grãos dos experimentos em rede de ensaio de cultivares de milho.

\section{CONCLUSÕES}

Os experimentos em rede de ensaio nacional de competição de cultivares de milho apresentam uma média precisão experimental, independente do ciclo dos materiais avaliados.

Para obter menores valores do quadrado médio do erro em experimentos de rede de ensaio nacional de milho, devem-se utilizar tratamentos (híbridos de milho) mais homogêneos para peso de espigas, rendimento de grãos, alturas da planta e de inserção da primeira espiga. 
Tabela 3 - Coeficientes de correlação parcial e de Pearson, devidos aos efeitos direto e indireto das variáveis observadas nos 98 experimentos de cultivares de milho de ciclo normal, sobre a estatística quadrado médio do erro (QMe). Santa Maria - RS, 2000.

\begin{tabular}{|c|c|c|c|c|c|c|c|c|c|c|c|c|c|c|c|c|c|c|}
\hline \multirow{2}{*}{ Efeito } & \multicolumn{18}{|c|}{ Variável $^{1}$} \\
\hline & Mpesp & Vpesp & Mpg & Vpg & Mfl & Vfl & Maltpl & Valtpl & Maltesp & Valtesp & Mpac & Vpac & Mpqb & Vpqb & Mef & Vef & Mnesp & Vnesp \\
\hline Direito & $0,32 *$ & $0,72^{*}$ & $0,29 *$ & $-0,03$ & $-0,12$ & $-0,62^{*}$ & $-0,60^{*}$ & $0,34 *$ & $-0,40 *$ & $0,37^{*}$ & 0,02 & 0,05 & 0,02 & 0,02 & 0,00 & 0,00 & 0,00 & 0,00 \\
\hline Mpesp & - & $-0,00$ & 0,15 & $-0,02$ & 0,08 & $-0,03$ & $-0,13$ & $-0,07$ & $-0,10$ & $-0,04$ & $-0,01$ & $-0,01$ & $-0,01$ & 0,00 & $-0,01$ & 0,00 & 0,00 & 0,00 \\
\hline Vpesp & $-0,01$ & - & 0,08 & $0,40^{*}$ & 0,10 & $-0,07$ & $-0,07$ & $-0,10$ & $-0,05$ & $-0,08$ & $-0,04$ & $-0,03$ & $-0,03$ & 0,00 & $-0,02$ & 0,00 & 0,00 & 0,00 \\
\hline Mpg & 0,14 & 0,03 & - & 0,00 & 0,04 & $-0,03$ & $-0,12$ & $-0,06$ & $-0,08$ & $-0,03$ & $-0,02$ & $-0,01$ & $-0,01$ & 0,00 & $-0,01$ & 0,00 & 0,00 & 0,00 \\
\hline Vpg & 0,00 & $-0,02$ & $-0,00$ & - & 0,01 & 0,00 & 0,01 & 0,01 & 0,01 & 0,00 & 0,00 & 0,00 & 0,00 & 0,00 & 0,00 & 0,00 & 0,00 & 0,00 \\
\hline Mfl & 0,01 & 0,01 & $-0,02$ & 0,04 & - & $-0,01$ & $-0,07$ & $-0,10$ & 0,13 & 0,04 & 0,05 & 0,00 & 0,08 & 0,00 & 0,02 & 0,00 & 0,00 & 0,00 \\
\hline Vfl & 0,06 & 0,06 & 0,05 & 0,06 & $-0,04$ & - & $-0,13$ & $-0,09$ & 0,01 & 0,02 & 0,01 & 0,01 & 0,01 & 0,00 & 0,00 & 0,00 & 0,00 & 0,00 \\
\hline Maltpl & 0,05 & 0,00 & 0,05 & 0,07 & $-0,01$ & $-0,12$ & - & $-0,01$ & $-0,04$ & $-0,13$ & 0,06 & 0,05 & 0,03 & 0,00 & 0,03 & 0,00 & 0,00 & 0,00 \\
\hline Valtpl & 0,07 & 0,09 & 0,07 & 0,08 & $-0,00$ & $-0,27$ & $-0,13$ & - & $-0,09$ & $-0,08$ & 0,02 & 0,01 & 0,01 & 0,00 & 0,01 & 0,00 & 0,00 & 0,00 \\
\hline Maltesp & 0,13 & 0,14 & 0,11 & 0,12 & 0,05 & 0,01 & $-0,07$ & $-0,11$ & - & $-0,13$ & 0,02 & 0,02 & 0,01 & 0,00 & 0,01 & 0,00 & 0,00 & 0,00 \\
\hline Valtesp & 0,05 & 0,04 & 0,05 & 0,04 & 0,05 & 0,01 & $-0,08$ & $-0,09$ & $-0,12$ & - & 0,00 & 0,01 & 0,00 & 0,00 & 0,00 & 0,00 & 0,00 & 0,00 \\
\hline Mpac & 0,01 & 0,01 & 0,01 & 0,01 & 0,02 & 0,00 & 0,02 & 0,01 & 0,01 & 0,00 & - & $-0,01$ & $-0,02$ & 0,00 & 0,00 & 0,00 & 0,00 & 0,00 \\
\hline Vpac & $-0,02$ & $-0,02$ & $-0,02$ & $-0,02$ & $-0,03$ & $-0,01$ & $-0,04$ & $-0,02$ & $-0,03$ & $-0,01$ & 0,09 & - & 0,01 & 0,00 & $-0,00$ & 0,00 & 0,00 & 0,00 \\
\hline Mpqb & $-0,03$ & $-0,03$ & $-0,02$ & $-0,02$ & $-0,03$ & $-0,01$ & $-0,04$ & $-0,02$ & $-0,03$ & $-0,01$ & 0,08 & 0,02 & - & 0,00 & $-0,00$ & 0,00 & 0,00 & 0,00 \\
\hline Vpqb & 0,00 & 0,00 & 0,00 & 0,00 & 0,00 & 0,00 & 0,00 & 0,00 & 0,00 & 0,00 & 0,00 & 0,00 & 0,00 & - & 0,00 & 0,00 & 0,00 & 0,00 \\
\hline Mef & 0,00 & 0,00 & 0,00 & 0,00 & 0,00 & 0,00 & 0,00 & 0,00 & 0,00 & 0,00 & 0,00 & 0,00 & 0,00 & 0,00 & - & 0,00 & 0,00 & 0,00 \\
\hline Vef & 0,00 & 0,00 & 0,00 & 0,00 & 0,00 & 0,00 & 0,00 & 0,00 & 0,00 & 0,00 & 0,00 & 0,00 & 0,00 & 0,00 & 0,00 & - & 0,00 & 0,00 \\
\hline Mnesp & 0,00 & 0,00 & 0,00 & 0,00 & 0,00 & 0,00 & 0,00 & 0,00 & 0,00 & 0,00 & 0,00 & 0,00 & 0,00 & 0,00 & 0,00 & 0,00 & - & 0,00 \\
\hline Vnesp & 0,00 & 0,00 & 0,00 & 0,00 & 0,00 & 0,00 & 0,00 & 0,00 & 0,00 & 0,00 & 0,00 & 0,00 & 0,00 & 0,00 & 0,00 & 0,00 & 0,00 & - \\
\hline Pearson & $0,14^{*}$ & $0,29 *$ & $0,19 *$ & $0,30^{*}$ & $-0,19 *$ & $-0,08$ & $-0,38^{*}$ & $0,20^{*}$ & $0,25^{*}$ & $0,28^{*}$ & $-0,05$ & 0,04 & $-0,03$ & 0,00 & $-0,02$ & 0,00 & 0,00 & 0,00 \\
\hline
\end{tabular}

${ }^{1} \mathrm{M}$ e V indicam, respectivamente, média e variância das variáveis: peso de espiga, peso de grãos, dias até florescimento, altura de planta, altura de inserção da primeira espiga, número de plantas acamadas, número de plantas quebradas, estande final e número de espigas;

*: Significativo em nível de $5 \%$ de probabilidade de erro.

\section{REFERÊNCIAS BIBLIOGRÁFICAS}

BANZATTO, D.A., KRONKA, S.N. Experimentação agrícola. 3.ed. Jaboticabal : FUNEP, 1995. 247p.

CONAGIN, A., IGUE, T., NAGAI, V. Tabelas para determinação do número de repetições no planejamento de experimentos. Campinas : Instituto Agronômico, 1995. 17p. (Boletim Científico, 34).

COSTA NETO, P.L.O. Estatística. São Paulo : Edgard Blücher, 1977. 264p.

CRUZ, C.D. Programa GENES: aplicativo computacional em genética e estatística. Viçosa : UFV, 1997. 442p.

CRUZ, C.D., REGAZZI, A.J. Modelos biométricos aplicados ao melhoramento genético. Viçosa : UFV, 1994. 390p.

EMBRAPA. Centro Nacional de Pesquisa de Milho e Sorgo. Ensaio Nacional de cultivares de Milho: resultados do ano agrícola 1996/1997. Sete Lagoas: EMBRAPA/CNPMS/ ABRASEM, 1997. 248p.

FEDERER, W.T. Experimental design. 3.ed. Nova York : Oxford \& IBH, 1977. 591p.

GOMES, F.P. A importância do número de repetições nos experimentos. Revista de Agricultura, Piracicaba, v.69, n.3, p.243-245, 1994.

HINKELMANN, K., KEMPTHORNE, O. Design and analysis of experiments. Nova York : John Wiley, 1994. v.1. 495p.
LOPES, S.J. Avaliação do efeito de diferentes formas de adubação sobre a precisão de ensaios de cultivares de milho. Santa Maria-RS, 1993. 72p. Dissertação (Mestrado em Agronomia) - Centro de Ciências Rurais, Universidade Federal de Santa Maria

LOPES, S.J., STORCK, L. A precisão experimental para diferentes manejos na cultura do milho. Ciência Rural, Santa Maria, v.25, n.1, p.49-53, 1995.

LÚCIO, A.D. Parâmetros da precisão experimental das principais culturas anuais do Estado do Rio Grande do Sul. Santa Maria-RS, 1997. 62p. Dissertação (Mestrado em Agronomia) - Centro de Ciências Rurais, Universidade Federal de Santa Maria.

NETER, J., WASSERMAN, W. Applied linear statistical models. 5.ed. Illinois : Richard D. Irwin, 1999. 842p.

RECOMENDAÇÕES técnicas para o cultivo do milho. Empresa Brasileira de Pesquisa Agropecuária. Brasília : EMBRAPA - SPI, 1993. 204p.

STEEL, R.G.D., TORRIE, J.H., DICKEY, D.A. Principles and procedures of statistics: a biometrical approach. 3.ed. Nova York : McGraw-Hill, 1996. 672p.

STORCK, L., GARCIA, D.C., LOPES, S.J., et $\boldsymbol{a l}$. Experimentação vegetal. Santa Maria : Universitária, 2000. $198 \mathrm{p}$

VENCOVSKY, R., BARRIGA, P. Genética biométrica no fitomelhoramento. Ribeirão Preto : Revista Brasileira de Genética, 1992. 496p.

Ciência Rural, v. 31, n. 5, 2001. 